\title{
Alcoves et $p$-rang des variétés abéliennes
}

\author{
B.C. Ngô, A. Genestier \\ dédié au Professeur Ngô Huy Cân
}

\begin{abstract}
Résumé
On étudie la relation entre le $p$-rang des variétés abéliennes en caractéristique $p$ et la stratification de Kottwitz-Rapoport de la fibre spéciale en $p$ de l'espace de module des variétés abéliennes principalement polarisées avec structure de niveau de type Iwahori en $p$. En particulier, on démontre la densité du lieu ordinaire dans cette fibre spéciale.
\end{abstract}

\section{Alcoves and $p$-rank of abelian varieties.}

We study the relation between the $p$-rank of abelian varieties in characteristic $p$ and the Kottwitz-Rapoport's stratification of the special fiber modulo $p$ of the moduli space of principally polarized abelian varieties with Iwahori type level structure on $p$. In particular, the density of the ordinary locus in that special fiber is proved.

\section{Introduction}

Dans [10], de Jong a étudié la mauvaise réduction modulo $p$ de l'espace de modules $\mathcal{A}$ des variétés abéliennes de dimension $n$, principalement polarisées et avec structure de niveau $\Gamma_{0}(p)$ en $p$. En utilisant le théorème de GrothendieckMessing sur les déformations de schémas abéliens, il a ramené l'étude des singularités de cette mauvaise réduction à l'étude de celles d'un autre problème de modules $\mathcal{M}$ défini pûrement en termes d'algèbre linéaire. Il s'agit là d'un cas particulier des modèles locaux que Rapoport et Zink [12 ont associé aux variétés de Shimura de type PEL.

L'une des propriétés fondamentales de $\mathcal{M}$ est que sa fibre spéciale peut être naturellement plongée dans l'ind-schéma de drapeaux affines (au sens de [1]) du groupe symplectique ( $c f$. [9], [3], [5] et aussi [2]). De ce fait, elle est réunion (finie, indicée par une partie finie KR du groupe de Weyl affine) d'orbites sous l'action du sous-groupe d'Iwahori. Ceci induit sur la fibre spéciale $\mathcal{A}$ une certaine stratification. Le propos de cette note est de démontrer que cette 
stratification est plus fine que celle définie par le $p$-rang des variétés abéliennes. On obtiendra en particulier que les strates ordinaires sont celles associées aux éléments de KR qui sont des translations. En conjonction avec un théorème de Kottwitz-Rapoport, ceci démontre donc que les strates ordinaires sont denses dans la fibre spéciale de $\mathcal{A}$, ce qui répond à une question posée par de Jong dans [10].

En ce qui concerne l'organisation du papier, les trois premières sections où nous rappelons le contexte du problème, sont connues des experts. Seule la dernière section est donc originale -signalons toutefois que U. Goertz a aussi trouvé la formule pour le $p$-rang, alors que circulait déjà une première version de cet article dans laquelle il n'était question que du problème de densité.

Nous exprimons notre gratitude à $M$. Rapoport duquel nous avons beaucoup appris au sujet de la mauvaise réduction des variétés de Shimura et qui par ses commentaires [11] a contribué à améliorer cet article. Nous remercions T. Haines pour avoir lu attentivement le manuscript et pour de nombreuses discussions sur ce sujet. Nous remercions aussi R. Kottwitz et J. Tilouine pour l'interêt qu'ils ont porté à ce travail.

\section{Rappels sur le modèle local}

On fixe un entier $n \geq 1$, un nombre premier $p$ et un entier $N$ premier à $p$. On note $T=\operatorname{Spec}\left(\mathbb{Z}_{p}\right), \eta$ le point générique de $T$ et $s$ son point fermé.

On considère le foncteur $\mathcal{A}$ qui associe à toute $\mathbb{Z}_{p}$-algèbre $R$, l'ensemble des classes d'isomorphisme de

$$
A=\left(A_{0} \stackrel{\alpha}{\longrightarrow} A_{1} \stackrel{\alpha}{\longrightarrow} \cdots \stackrel{\alpha}{\longrightarrow} A_{n}, \lambda_{0}, \lambda_{n}, \iota_{N}\right)
$$

où

- $A_{0} \rightarrow A_{1} \rightarrow \cdots \rightarrow A_{n}$ est une suite d'isogénies de schémas abéliens de dimension $n$ sur $S=\operatorname{Spec}(R)$ tels que les $\operatorname{Ker}\left(A_{i} \rightarrow A_{i+1}\right)$ sont des schémas en groupes finis et plats de rang $p$ sur $S$

- $\lambda_{0}$ et $\lambda_{n}$ sont des polarisations principales de $A_{0}$ et $A_{n}$ telles que le composé des flèches partant de $A_{i}$ et revenant à $A_{i}$ après avoir fait le tour du diagramme

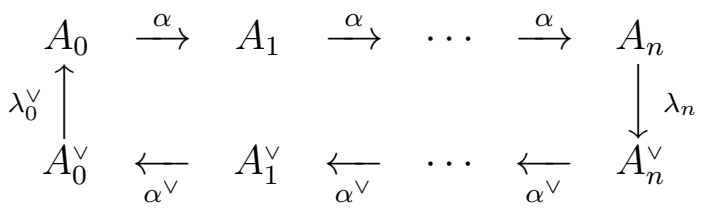

est égal à $p \cdot \operatorname{Id}_{A_{i}}$ pour tout $i=0, \ldots, g$. Ici on a dégigné par $A_{i}^{\vee}$ le schéma abélien dual à $A_{i}$. 
- $\iota_{N}$ est un isomorphisme symplectique $\iota_{N}: A_{0}[N] \rightarrow(\mathbb{Z} / N \mathbb{Z})^{2 n}$ pour une forme symplectique fixée sur $(\mathbb{Z} / N \mathbb{Z})^{2 n}$

Pour $N$ assez grand, ce foncteur est représentable par un $T$-schéma $\mathcal{A}$ ayant des singularités dans la fibre spéciale. Rappelons la construction de de Jong et de Rapoport-Zink du modèle local de ces singularités.

Pour tout $i=0, \ldots, n$, les ingrédients principaux de la construction du modèle local sont $M_{i}=\mathrm{R}^{1} a_{i *}\left(\Omega_{A_{i} / S}^{\bullet}\right)$ et $\omega_{i}=a_{i, *} \Omega_{A_{i} / S}^{1}$ où $a_{i}$ est le morphisme structurel $a_{i}: A_{i} \rightarrow S$.

Les $M_{i}$ sont des $\mathcal{O}_{S}$-module localement libre de rang $2 n$ qui viennent avec :

- les homomorphismes de $\mathcal{O}_{S}$-modules

$$
M_{n} \stackrel{\alpha}{\longrightarrow} M_{n-1} \stackrel{\alpha}{\longrightarrow} \cdots \stackrel{\alpha}{\longrightarrow} M_{0}
$$

induits des isogénies $\alpha: A_{i} \rightarrow A_{i+1}$.

- les formes symplectiques non dégénérées

$$
q_{0}: M_{0} \otimes_{\mathcal{O}_{S}} M_{0} \rightarrow \mathcal{O}_{S} \quad \text { et } \quad q_{n}: M_{n} \otimes_{\mathcal{O}_{S}} M_{n} \rightarrow \mathcal{O}_{S}
$$

induites par les polarisations principales $\lambda_{0}$ et $\lambda_{n}$ sur $A_{0}$ et $A_{n}$.

Notons $M(A)$ la donnée

$$
M(A)=\left(M_{n} \stackrel{\alpha}{\longleftarrow} M_{g-1} \stackrel{\alpha}{\longleftarrow} \cdots \stackrel{\alpha}{\longleftarrow} M_{0}, q_{0}, q_{n}\right) .
$$

Elle vérifie les conditions suivantes (voir loc. cit. prop. 3.1)

- Les $\operatorname{Coker}\left(M_{i} \stackrel{\delta}{\longrightarrow} M_{i+1}\right)$ sont des $\mathcal{O}_{S} / p \mathcal{O}_{S}$-module localement libres de rang 1 .

- Pour tout $i=0, \ldots, n$, le composé des flèches partant de $M_{i}$ et revenant à $M_{i}$ après avoir fait le tour du diagramme

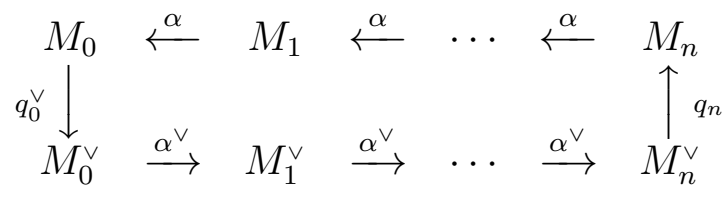

est égal à la multiplication par $p \cdot \operatorname{Id}_{M_{i}}$. Ici on a posé $M_{i}^{\vee}=\operatorname{Hom}\left(M_{i}, \mathcal{O}_{S}\right)$.

On appelle la donnée $M$ de $\mathcal{O}_{S}$-modules localement libres de rang $2 n$ munis des homomorphismes $\mathcal{O}_{S^{-}}$linéaires $\alpha$

$$
\left(M_{0} \stackrel{\alpha}{\longleftarrow} M_{1} \stackrel{\alpha}{\longleftarrow} \cdots \stackrel{\alpha}{\longleftarrow} M_{n}, q_{0}, q_{n}\right)
$$

et des formes symplectiques $q_{0}$ et $q_{n}$ vérifiant les deux dernières propriétés, un système $\mathrm{Sp}$ de $\mathcal{O}_{S}$-modules de type $\Gamma_{0}(p)$. Rappelons le lemme suivant, dû à de Jong (loc. cit. lemma 3.6; voir aussi [12], appendix to chapter 3 : normal forms of lattice chains pour un énoncé pus général). 
Lemme 1.1 (de Jong) Deux systèmes $\mathrm{Sp}$ de $\mathcal{O}_{S}$-modules de type $\Gamma_{0}(p)$ arbitraires $M$ et $M^{\prime}$ sont localement isomorphes pour la topologie de Zariski de S. Si I est un idéal de carré nul de $\mathcal{O}_{S}$, si $S^{\prime}$ est le sous-schéma fermé de $S$ défini par $I$, tout isomorphisme entre les restrictions de $M$ et $M^{\prime}$ à $S^{\prime}$ se relève en un isomorphisme entre $M$ et $M^{\prime}$.

Il existe un système $\mathrm{Sp}$ de $\mathcal{O}_{T}$-modules de type $\Gamma_{0}(p)$ standard, noté $V$ dont on précisera la construction dans la section suivante. Une conséquence immédiate du lemme de de Jong est que le foncteur $S \mapsto \operatorname{Aut}\left(V \otimes_{\mathcal{O}_{T}} \mathcal{O}_{S}\right)$ est représentable par un schéma en groupes lisse de type fini sur $T$.

On considère le foncteur $\mathcal{W}$ qui associe à tout $T$-schéma $S$ l'ensemble des couples $(A, \iota)$ où $A \in \mathcal{A}(S)$ et où $\iota$ est un isomorphisme

$$
\iota: M(A) \rightarrow V \otimes_{\mathcal{O}_{T}} \mathcal{O}_{S}
$$

de système $\mathrm{Sp}$ de $\mathcal{O}_{S}$-modules de type $\Gamma_{0}(p)$. Le foncteur d'oubli induit un morphisme $\pi: \mathcal{W} \rightarrow \mathcal{A}$. L'énoncé suivant résulte alors également du lemme de de Jong rappelé ci-dessus.

Proposition 1.2 Le morphisme $\pi: \mathcal{W} \rightarrow \mathcal{A}$ est un torseur sous le schéma en groupes $\operatorname{Aut}(V)$. En particulier, c'est un morphisme représentable, séparé lisse et surjectif.

Maintenant pour chaque $i, M_{i}=\mathrm{R}^{1} a_{i *} \Omega_{A_{i} / S}^{\bullet}$ contient un $\mathcal{O}_{S^{-s o u s}-m o d u l e}$

$$
\omega_{i}=a_{i *} \Omega_{A_{i} / S}^{1} \subset M_{i}
$$

qui est localement un facteur direct de rang $n$. Ces sous-modules $\omega_{i}$ vérifient les conditions suivantes

$-\alpha\left(\omega_{i+1}\right) \subset \omega_{i}$,

- $\omega_{0}$ et $\omega_{n}$ sont totalement isotropes par rapport à $q_{0}$ et $q_{n}$ respectivement.

Considérons le foncteur $\mathcal{M}$ qui associe à chaque $T$-schéma $S$ l'ensemble des

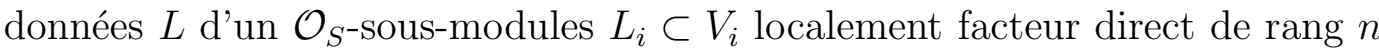
pour $i=0, \ldots, n$ tels que

$-\alpha\left(L_{i+1}\right) \subset L_{i}$

- $L_{0}$ et $L_{n}$ sont totalement isotropes par rapport à $q_{0}$ et $q_{n}$ respectivement.

La donnée d'un $S$-point $A \in \mathcal{A}(S)$ et d'un isomorphisme $\iota: M(A) \rightarrow V \otimes$ $\mathcal{O}_{S}$ de systèmes $\operatorname{Sp}$ de $\mathcal{O}_{S}$-modules de type $\Gamma_{0}(p)$, définit un point $L \in \mathcal{M}(S)$ par la règle $L_{i}=\iota\left(\omega_{i}\right)$. On obtient ainsi un morphisme $f: \mathcal{W} \rightarrow \mathcal{M}$.

En utilisant la théorie de Grothendieck-Messing des déformations de schémas abéliens, de Jong [10] et Rapoport et Zink [12] ont démontré le théorème suivant. 
Théorème 1.3 (de Jong, Rapoport-Zink) Le morphisme $f: \mathcal{W} \rightarrow \mathcal{M}$ est un morphisme lisse.

D'après [2], on sait de plus que le morphisme $f: \mathcal{W} \rightarrow \mathcal{M}$ est surjectif.

\section{Système standard}

Soit $\mathbb{A}_{T}^{1}=\operatorname{Spec}\left(\mathbb{Z}_{p}[t]\right)$ la droite affine au-dessus de $T=\operatorname{Spec}\left(\mathbb{Z}_{p}\right)$. Soient $\mathcal{V}_{0}, \ldots, \mathcal{V}_{2 n}$ des $\mathcal{O}_{\mathbb{A}_{T}^{1}}$-modules libres de rang $2 n$. Soient $q_{0}: \mathcal{V}_{0} \otimes \mathcal{V}_{0} \rightarrow \mathcal{O}_{\mathbb{A}_{T}^{1}}$ et $q_{n}: \mathcal{V}_{n} \otimes \mathcal{V}_{n} \rightarrow \mathcal{O}_{\mathbb{A}_{T}^{1}}^{T}$ les formes symplectiques non-dégénérées associées à la matrice alternée

$$
J=\left(\begin{array}{cc}
0 & K_{n} \\
-K_{n} & 0
\end{array}\right)
$$

où $K_{n}$ est la matrice $n \times n$ avec que des 1 sur l'anti-diagonale et que des 0 ailleurs. Soit $\alpha: \mathcal{V}_{i+1} \rightarrow \mathcal{V}_{i}$ l'homomorphisme de $\mathcal{O}_{\mathbb{A}_{T}^{1}}$-modules défini par

$$
\alpha=\left(\begin{array}{cc}
0 & \operatorname{Id}_{2 n-1} \\
t-p & 0
\end{array}\right) .
$$

Notons $x_{0}: T \rightarrow \mathbb{A}_{T}^{1}$ et $x_{p}: T \rightarrow \mathbb{A}_{T}^{1}$ les sections définies par $t=0$ et $t=p$ respectivement. Puisque $\alpha^{2 n}=(t-p) \operatorname{Id}_{2 n}$, les conoyaux de $\alpha: \mathcal{V}_{i+1} \rightarrow \mathcal{V}_{i}$ sont supportées par la section $x_{p}$.

Posons maintenant $V_{i}=x_{0}^{*} \mathcal{V}_{i}$ et désignons aussi par $\alpha, q_{0}, q_{n}$ les restrictions de $\alpha, q_{0}, q_{n}$ à $x_{0}$. On vérifie sans peine l'assertion suivante.

Proposition 2.1 La donnée $V=\left(V_{0} \stackrel{\alpha}{\longleftarrow} V_{1} \stackrel{\alpha}{\longleftarrow} \cdots \stackrel{\alpha}{\longleftarrow} V_{n}, q_{0}, q_{n}\right)$ forme un système Sp de $\mathcal{O}_{T}$-modules de type $\Gamma_{0}(p)$.

Il sera commode d'identifier $\mathcal{V}_{i}$ avec son image par l'homomorphisme injectif $\alpha^{i}: \mathcal{V}_{i} \rightarrow \mathcal{V}_{0}$ de sorte que la suite des morphismes

$$
\mathcal{V}_{0} \stackrel{\alpha}{\longleftarrow} \mathcal{V}_{1} \stackrel{\alpha}{\longleftarrow} \ldots \stackrel{\alpha}{\longleftarrow} \mathcal{V}_{2 n}
$$

puisse s'identifier à une suite d'inclusions de $\mathcal{O}_{\mathbb{A}_{T}^{1}}$-modules

$$
\mathcal{V}_{0} \supset \mathcal{V}_{1} \supset \cdots \supset \mathcal{V}_{2 n-1} \supset \mathcal{V}_{2 n}=\mathcal{V}_{0}\left[-x_{p}\right]
$$

Après cette identification, la forme $(t-p)^{-1} K$ définit des accouplements parfaits $\mathcal{V}_{i} \otimes_{\mathcal{O}_{\mathbb{A}_{T}^{1}}^{1}} \mathcal{V}_{2 n-i} \rightarrow \mathcal{O}_{\mathbb{A}_{T}^{1}}$ qui induisent des accouplements parfaits $V_{i} \otimes_{\mathcal{O}_{T}}$ $V_{2 n-i} \rightarrow \mathcal{O}_{T}^{T}$. De plus, l'inclusion $\mathcal{V}_{i} \subset \mathcal{V}_{i-1}$ est duale à $\mathcal{V}_{2 n-i+1} \subset \mathcal{V}_{2 n-i}$. On est maintenant en mesure de réécrire le problème de modules de $\mathcal{M}$ en termes de chaînes de réseaux.

Rappelons que le foncteur modèle local $\mathcal{M}$ associe à chaque $T$-schéma $S$ l'ensemble des données $L$ d'un $\mathcal{O}_{S}$-sous-modules $L_{i} \subset V_{i} \otimes_{\mathcal{O}_{T}} \mathcal{O}_{S}$ localement facteur direct de rang $n$ pour $i=0, \ldots, n$ tels que 
$-\alpha\left(L_{i+1}\right) \subset L_{i}$

- $L_{0}$ et $L_{n}$ sont totalement isotropes par rapport à $q_{0}$ et $q_{n}$ respectivement.

En utilisant la dualité entre $V_{i}$ et $V_{2 n-i}$, on obtient pour tout $i=n+1, \ldots, 2 n$ un sous- $\mathcal{O}_{S^{-}}$module $L_{i} \subset V_{i}$ qui est dual à $L_{2 n-i} \subset V_{2 n-i}$.

Notons que la donnée d'un sous- $\mathcal{O}_{S}$-module localement facteur direct $L_{i} \subset$ $V_{i} \otimes_{\mathcal{O}_{T}} \mathcal{O}_{S}$ est équivalente à la donnée d'un $\mathcal{O}_{\mathbb{A}_{S}^{1}}$-module localement libre de rang $2 n$ munie des modifications

$$
\mathcal{V}_{i} \otimes_{\mathcal{O}_{T}} \mathcal{O}_{S}\left[-x_{0}\right] \subset \mathcal{L}_{i} \subset \mathcal{V}_{i} \otimes_{\mathcal{O}_{T}} \mathcal{O}_{S}
$$

si bien que la donnée d'un $S$-point de $\mathcal{M}$ est maintenant équivalente à celle d'un diagramme d'inclusions de $\mathcal{O}_{\mathbb{A}_{S}^{1}}$-modules

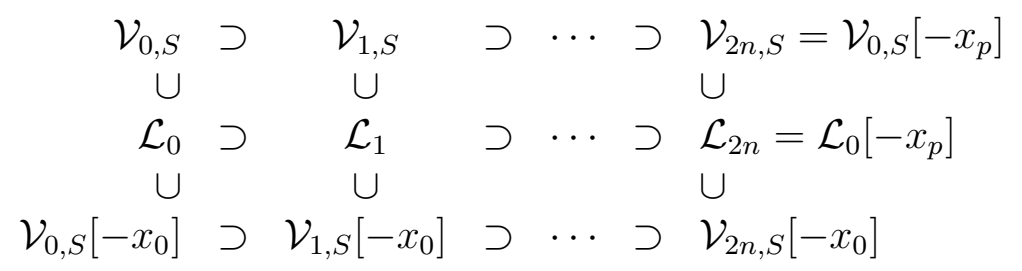

telle que pour tout $i$, les deux modules $\mathcal{L}_{i}$ et $\mathcal{L}_{2 n-i}$ sont en dualité par rapport à la forme $(t-p)^{-1} K$ et que $\mathcal{L}_{i} / \mathcal{V}_{i . S}\left[-x_{0}\right]$ est un sous- $\mathcal{O}_{S}$-module localement facteur direct de rang $n$ de $V_{i, S}=\mathcal{V}_{i, S} / \mathcal{V}_{i, S}\left[-x_{0}\right]$

Cette nouvelle présentation du problème de modules $\mathcal{M}$ semble nettement plus compliquée que la précédente. En contre partie, elle met en lumière le fait important suivant (pour lequel nous renvoyons à l'article de Kottwitz et Rapoport [9], à celui de Goertz [3] et à celui de Haines et du premier auteur [5]; voir aussi dans [2] la preuve de la surjectivité du morphisme $f: \mathcal{W} \rightarrow \mathcal{M}$ ).

Proposition 2.2 La fibre spéciale $\mathcal{M}_{s}$ est un sous-schéma fermé de l'indschéma de drapeaux affine de $\operatorname{GSp}(2 n)$ (au sens de [1]).

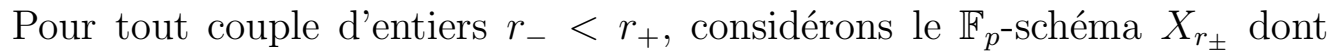
l'ensemble des $S$-points, pour toutes $\mathbb{F}_{p}$-algèbre $S$, est l'ensemble des diagrammes d'inclusions de $\mathcal{O}_{\mathbb{A}_{S}^{1}}$-modules

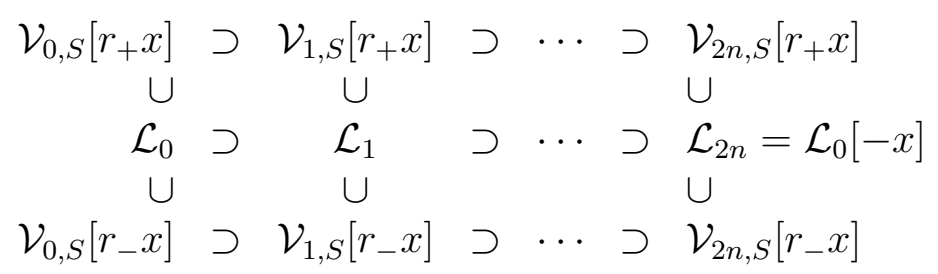

où $x=x_{0}=x_{p}$ en caractéristique $p$, et tels que $\mathcal{L}_{i}$ et $\mathcal{L}_{2 n-i}$ sont duaux par rapport à la forme $t^{r_{+}+r_{-}} K$ et que $\mathcal{L}_{i} / \mathcal{V}_{i, S}\left[r_{-} x\right]$ est un sous- $\mathcal{O}_{S}$-module 
localement facteur direct de rang $n\left(r_{+}-r_{-}\right)$de $\mathcal{V}_{i, S}\left[r_{+} x\right] / \mathcal{V}_{i, S}\left[r_{-} x\right]$. Visiblement $\mathcal{M}_{s}=X_{0,-1}$. Les $X_{r_{ \pm}}$sont tous des $\mathbb{F}_{p}$-schémas projectifs et forment un système inductif avec $r_{+} \rightarrow \infty$ et $r_{-} \rightarrow-\infty$.

$\mathrm{Si}$ on localise en dehors de la section $x$, les inclusions dans le diagramme deviennent toutes des égalités. Notons $\mathcal{O}_{x}$ l'anneau local complété de $\mathbb{F}_{p}[t]$ en $t=0$. On peut identifier les complétés en $x$ de $\mathcal{V}_{i, \mathbb{F}_{p}}$ avec

$$
\mathcal{V}_{i, \mathbb{F}_{p}, x}=\bigoplus_{j=1}^{i} \mathcal{O}_{x} e_{j} \oplus \bigoplus_{j=i+1}^{2 n} t \mathcal{O}_{x} e_{j}
$$

où $e_{1}, \ldots, e_{2 n}$ est la base standard. Par le théorème de recollement formel de Beauville et Laszlo [ [G], la donnée d'un $\mathbb{F}_{p}$-point de $\mathcal{M}_{r_{ \pm}}$est équivalente à la donnée d'une chaîne périodique de réseaux

$$
\mathcal{L}_{0, x} \supset \mathcal{L}_{1, x} \supset \cdots \supset \mathcal{L}_{2 n, x}=t \mathcal{L}_{0, x}
$$

vérifiant la condition d'inclusion

$$
t^{-r_{+}} \mathcal{V}_{i, \mathbb{F}_{p}, x} \supset \mathcal{L}_{i, x} \supset t^{-r} \mathcal{V}_{i, \mathbb{F}_{p}, x}
$$

pour tout $i$ et la condition de dualité évidente. En prenant la limite quand $r_{ \pm} \rightarrow \pm \infty$ on obtient donc l'ind-schéma $X$ des drapeaux affines de GSp $(2 n)$

$$
X\left(\mathbb{F}_{p}\right)=\operatorname{GSp}\left(2 n, \mathbb{F}_{p}((t))\right) / I\left(\mathbb{F}_{p}\right)
$$

où $I$ est un groupe algébrique sur $\mathbb{F}_{p}$ dont les $\mathbb{F}_{p}$-points forment le sous-groupe d'Iwahori (opposé) standard de $\operatorname{GSp}\left(2 n, \mathbb{F}_{p}((t))\right)$-la mention "opposé" résulte de notre choix de travailler avec les chaînes descendantes.

En particuler, le ind-groupe $\operatorname{GSp}\left(2 n, \mathbb{F}_{p}((t))\right)$ agit sur $X$. L'action restreinte à $I$ laisse stable les $X_{r_{ \pm}}$et en particulier, $I$ agit sur $X_{0,-1}=\mathcal{M}_{s}$. Il existe en fait un homomorphisme canonique de groupes algébriques

$$
I \rightarrow \operatorname{Aut}\left(V_{s}\right)
$$

à travers lequel se factorise l'action de $I$ sur $\mathcal{M}_{s}$. Pour la vérification facile, mais fastidieuse, des assertions précédentes, nous renvoyons à [5].

\section{L'ensemble de Kottwitz-Rapoport}

D'après Iwahori-Marsumoto [8], on a la décomposition

$$
\operatorname{GSp}\left(2 n, \mathbb{F}_{p}((t))\right)=\bigsqcup_{w \in \widetilde{W}_{a}} I\left(\mathbb{F}_{p}\right) w I\left(\mathbb{F}_{p}\right)
$$


où $\widetilde{W}_{a}$ est le groupe de Weyl affine étendu du groupe $\operatorname{GSp}(2 n)$. Cette décomposition se traduit géométriquement en une décomposition cellulaire $X=\bigsqcup_{w \in \widetilde{W}_{a}} X_{w}$ avec $X_{w}=\mathbb{A}^{\ell(w)}$ où $\ell(w)$ est la fonction longueur dont on rappellera la définition juste après. Du simple fait que $\mathcal{M}_{s}$ est un sous-schéma projectif et $I$-équivariant de $X$, on a

$$
\mathcal{M}_{s}=\bigsqcup_{w \in \operatorname{KR}(\mu)} X_{w}
$$

où $\mathrm{KR}(\mu)$ est un certain sous-ensemble fini de $\tilde{W}_{a}$ que Kottwitz et Rapoport appellent l'ensemble des éléments $\mu$-permissibles. Pour rappeler la définition combinatoire, due à Kottwitz et Rapoport [9] de cet ensemble fini, il nous faut faire quelques rappels sur le groupe de Weyl affine.

Soit $G$ un groupe semi-simple déployé défini sur $\mathbb{Z}_{p}, T$ un tore maximal déployé de $T, W=\operatorname{Nor}(T) / T$ le groupe de Weyl fini associé. Notons $Q$ le $\mathbb{Z}$-module engendré par les racines de $G$ dans l'espace vectoriel réel $X_{\mathbb{R}}=$ $\operatorname{Hom}\left(T, \mathbb{G}_{m}\right) \otimes \mathbb{R}$. Notons $Q^{\vee}$ le $\mathbb{Z}$-module engendré par les coracines dans l'espace vectoriel réel dual $X_{\mathbb{R}}^{\vee}=\operatorname{Hom}\left(\mathbb{G}_{m}, T\right) \otimes \mathbb{R}$ et $P^{\vee}$ le réseau dual à $Q$.

Soient $W_{a}=W \ltimes Q^{\vee}$ le groupe de Weyl affine et $\widetilde{W}_{a}=W \ltimes P^{\vee}$ le groupe de Weyl affine étendu. Rappelons que le groupe $W_{a}$ est un groupe de Coxeter de sorte qu'il muni d'un ordre partiel - celui de Bruhat- et d'une fonction longueur $\ell: W_{a} \rightarrow \mathbb{N}$. Une racine $\alpha$ de $G$ et un entier $k \in \mathbb{Z}$ définissent un mur affine

$$
H_{\alpha, k}=\left\{x \in X_{\mathbb{R}}^{\vee} \mid\langle\alpha, x\rangle=k\right\}
$$

Le complémentaire dans $X_{\mathbb{R}}^{\vee}$ de la réunion de tous les murs affines se décompose en composantes connexes qui sont appelées les alcoves.

Soient $\left(\alpha_{i}\right)_{i \in I}$ les racines simples associées au choix d'un sous-groupe de Borel standard, $\gamma$ la plus grande racine. Il existe une alcove, dite de base, qui est délimitée par les murs $H_{\alpha_{i}, 0}$ pour $i \in I$ et $H_{\gamma, 1}$. Pour chaque mur de l'alcove, il existe un unique sommet, dit opposé, qui ne lui appartient pas. Notons pour tout $i \in I, a_{i}$ le sommet opposé à $H_{\alpha_{i}, 0}$ et notons $a_{0}$ le sommet opposé à $H_{\gamma, 1}$.

Le groupe $\widetilde{W}_{a}$ agit sur $X_{\mathbb{R}}^{\vee}$ en envoyant un mur affine sur un autre, de sorte qu'il agit sur l'ensemble des alcoves. Rappelons que l'action de $W_{a}$ sur l'ensemble des alcoves est simple et transitive, voir [7]. Il s'ensuit que $\widetilde{W}_{a}=$ $W_{a} \Omega$ où $\Omega$ est le fixateur de l' alcove de base qui est un sous-groupe fini. L'ordre de Bruhat et la fonction longueur s'étendent trivialement de $W_{a}$ à $\widetilde{W}_{a}$. Pour tous $w, w^{\prime} \in W_{a}, \tau, \tau^{\prime} \in \Omega$ on dit $w \tau \leq w^{\prime} \tau^{\prime}$ si et seulement si $w \leq w^{\prime}$ et $\tau=\tau^{\prime}$. On pose $\ell(w \tau)=\ell(w)$.

Soit $\mu \in Q^{\vee}$ un copoids minuscule de $G$. D'après Kottwitz et Rapoport, un élément $w \in \widetilde{W}_{a}$ est dit $\mu$-permissible si pour tout sommet $a$ de l'alcove de 
base, la différence $w(a)-a$ est conjuguée à $\mu$ par rapport à l'action de groupe de Weyl fini $W$.

Pour comprendre géométriquement cette condition, considérons le cas $\mathrm{GL}(d)$. Le groupe de Weyl affine étendu $\widetilde{W}_{a}(\mathrm{GL}(d))$ agit sur l'ensemble des alcoves de $\operatorname{PGL}(d)$ à travers l'homomorphisme canonique surjectif $\widetilde{W}_{a}(\mathrm{GL}(d)) \rightarrow \widetilde{W}_{a}(\operatorname{PGL}(d))$. Soit

$$
\mu=(\underbrace{1, \ldots, 1}_{r}, 0, \ldots, 0)
$$

un copoids minuscule de $\mathrm{GL}(d)$ et notons aussi par $\mu$ le copoids minuscule induit de $\operatorname{PGL}(d)$. On choisit pour chaque $w \in \widetilde{W}(\mathrm{GL}(d))$ le représentant, noté aussi $\left.w \in \operatorname{GL}\left(d, \mathbb{F}_{q}((t))\right)\right)$ la matrice $d \times d$ avec exactement $d$ entrées non nulles lesquelles sont de la forme $t^{s}$. Ce choix est caractérisé par la propriété que $w$ stabilise l'ensemble de vecteurs $t^{m} e_{j}$ où $\left\{e_{j}\right\}$ est la base standard.

Rappelons qu'un $\mathbb{F}_{p}$-point $\mathcal{L}$ de l'ind-schéma des drapeaux affines de $\mathrm{GL}(d)$ est un drapeau périodique de réseaux

$$
\mathcal{L}_{0, x} \supset \mathcal{L}_{1, x} \supset \cdots \supset \mathcal{L}_{d, x}=t \mathcal{L}_{0, x}
$$

dans $\mathbb{F}_{p}((t))^{d}$. Rappelons aussi qu'on a défini un drapeau standard $\mathcal{V}_{i, \mathbb{F}_{p}, x}$ dans la section 2 .

Proposition 3.1 Supposons que $\mathcal{L} \in X_{w}(k)$ pour un certain $w \in \widetilde{W}_{a}(\mathrm{GL}(d))$ avec $\operatorname{val}(\operatorname{det}(w))=r$. Alors, $w$ est $\mu$-permissible si et seulement si pour tout $i=0, \ldots, d-1$, on a $\mathcal{V}_{i, \mathbb{F}_{p}, x} \supset \mathcal{L}_{i} \supset t \mathcal{V}_{i, \mathbb{F}_{p}, x}$.

Démonstration. Par définition, I stabilise le drapeau de base $\mathcal{V}_{i, \mathbb{F}_{p}, x}$, si bien qu'il suffit de tester le cas $\mathcal{L}_{i, x}=w \mathcal{V}_{i, k, x}$. On doit démontrer que pour tout $i=0, \ldots, d-1$, les inclusions

$$
\mathcal{V}_{i, \mathbb{F}_{p}, x} \supset w \mathcal{V}_{i, x} \supset t \mathcal{V}_{i, \mathbb{F}_{p}, x}
$$

ont lieu si et seulement si $w\left(a_{i}\right)-a_{i} \in W \mu$, toujours sous l'hypothèse val $(\operatorname{det}(w))=$ $r$.

Du fait qu'on considère le cas linéaire, les sommets $a_{1}, \ldots, a_{d-1}$ de l'alcove de base sont les copoids fondamentaux. Notons $\mathrm{t}_{a_{i}}$ l'élément $a_{i}$ vu comment un élément de $\widetilde{W}_{a}$ et comme élément de $\operatorname{GL}(d, k((t)))$. On a $\mathcal{V}_{i, \mathbb{F}_{p}, x}=\mathrm{t}_{a_{i}} \mathcal{V}_{0, \mathbb{F}_{p}, x}$. Les inclusions

$$
\mathcal{V}_{i, \mathbb{F}_{p}, x} \supset w \mathcal{V}_{i, x} \supset t \mathcal{V}_{i, \mathbb{F}_{p}, x}
$$

ont lieu si et seulement si

$$
\mathrm{t}_{-a_{i}} w \mathrm{t}_{a_{i}} \in K \mu K
$$

où $K=\mathrm{GL}(n, k[[t]])$ est le stabilisateur de $\mathcal{V}_{0, k}$. Il s'ensuit que $\mathrm{t}_{-a_{i}} w \mathrm{t}_{a_{i}} \in y \mathrm{t}_{\mu} W$ pour un certain $\mu \in W$ d'où $w\left(a_{i}\right)-a_{i}=y \mu$ en appliquant $\mathrm{t}_{-a_{i}} w \mathrm{t}_{a_{i}}$ à 0 . 
Notons tout de suite que cette démonstration n'est valable que dans le cas linéaire. Dans le cas du groupe $\operatorname{GSp}(2 n)$ avec le copoids minuscule

$$
\mu=(\underbrace{1, \ldots, 1}_{n}, \underbrace{0, \ldots, 0}_{n})
$$

on déduit directement de la proposition précédente et de la description fonctorielle de $\mathcal{M}_{s}$ l'assertion suivante.

Corollaire 3.2 On a une décomposition cellulaire

$$
\mathcal{M}_{s}=\bigsqcup_{w \in \operatorname{KR}(\mu)} X_{w}
$$

où $\operatorname{KR}(\mu)$ est l'ensemble des éléments de $\widetilde{W}_{a}(\operatorname{GSp}(2 n))$ dont l'image dans $\widetilde{W}_{a}(\mathrm{GL}(2 n))$ est $\mu$-permissible.

Kottwitz et Rapoport ont démontré qu'en plus, $\mathrm{KR}(\mu)$ est exactement l'ensemble des $\mu$-permissibles dans $\operatorname{GSp}(2 n)$ car les éléments qui sont $\mu$-permissibles dans $\operatorname{GSp}(2 n)$ sont aussi ceux dont l'image est $\mu$-permissible dans GL(2n). Nous préférons la description du corollaire qui est moins élégant, mais qui colle automatiquement à la description fonctorielle de $\mathcal{M}_{s}$. Par ailleurs, comme cela a été montré dans [6], pour un $\mu$ non minuscule, il existe des éléments $\mu$ permissibles dans $\operatorname{GSp}(2 n)$ dont l'image n'est pas $\mu$-permissible dans GL( $2 n)$. Mais revenons au cas minuscule pour rappeller le résultat important suivant.

Théorème 3.3 (Kottwitz-Rapoport) Si $w \in \mathrm{KR}(\mu)$ alors il existe $\mathrm{y} \in$ $\mathrm{W}(\mathrm{GSp}(2 \mathrm{n}))$-le groupe de Weyl vectoriel de $\operatorname{GSp}(2 n)-$ tel que $w \leq \mathrm{t}_{\mathrm{y} \mu}$.

Les éléments translations $\mathrm{t}_{\mathrm{y} \mu}$ sont clairement $\mu$-permissibles parce que $\mathrm{t}_{\mathrm{y} \mu}\left(a_{i}\right)-$ $a_{i}=\mathrm{y} \mu$. Le théorème dit qu'ils sont exactement les éléments maximaux de $\mathrm{KR}(\mu)$. Cet énoncé a été généralisé par Haines et Ngô à tous les poids dominants de $\operatorname{GSp}(2 n)[6$.

\section{Alcoves et $p$-rang}

Revenons aux notations de la section 1 où on a construit un morphisme lisse $\operatorname{Aut}(V)$-équivariant $f: \mathcal{W} \rightarrow \mathcal{M}$ et aussi un morphisme $\pi: \mathcal{W} \rightarrow \mathcal{A}$ qui fait de $\mathcal{W}$ un $\operatorname{Aut}(V)$-torseur au-dessus de $\mathcal{A}$. La stratification en $\operatorname{Aut}(V)_{s}$-orbites de $\mathcal{M}_{s}$ définie dans la section 3

$$
\mathcal{M}_{s}=\bigsqcup_{w \in \operatorname{KR}(\mu)} X_{w}
$$


induit par image inverse une stratification $\mathcal{W}_{s}=\bigsqcup_{w \in \mathrm{KR}(\mu)} \mathcal{W}_{w}$ dont les strates $\mathcal{W}_{w}=f^{-1}\left(X_{w}\right)$ sont $\operatorname{Aut}(V)_{s}$-équivariantes. Du fait que $\mathcal{W}$ est un $\operatorname{Aut}(V)$ torseur au-dessus de $\mathcal{A}$, cette stratification se descend en une stratification de $\mathcal{A}_{s}$

$$
\mathcal{A}_{s}=\bigsqcup_{w \in \operatorname{KR}(\mu)} \mathcal{A}_{w}
$$

telle que $\mathcal{W}_{w}=\pi^{-1}\left(\mathcal{A}_{w}\right)$ pour tout $w \in \mathrm{KR}(\mu)$. Cette stratification jouit des propriétés très agréables, déduites de celles connues sur le modèle local, que nous énumérons ici pour des références ultérieures :

- Les strates $\mathcal{A}_{w}$ sont lisses de dimension $l(w)$ d'après le théorème de lissité de de Jong et Rapoport-Zink. Elles sont non vides d'après la surjectivité de ([2], proposition 1.3.2).

- La restriction du complexe de cycles proches $\mathrm{R} \Psi_{\mathcal{A}} \mathbb{Q}_{\ell}$ à chaque strate $\mathcal{A}_{w}$ est constante. En effet, du côté du modèle local $\mathcal{M}$, le complexe des cycles proches est constant sur les strates $\mathcal{M}_{w}$ parce qu'il est équivariant par rapport à l'action de $\operatorname{Aut}(V)$.

- La trace semi-simple de Frobenius agissant sur une fibre quelconque de $\left.\mathrm{R} \Psi_{\mathcal{A}} \mathbb{Q}_{\ell}\right|_{\mathcal{A}_{w}}$ est calculée par la fonction de Bernstein-Kottwitz, d'après Haines et Ngô [5]. On a par ailleurs une formule très explicite de cette fonction due à Haines [4] : pour tout $A \in \mathcal{A}_{w}\left(\mathbb{F}_{q}\right)$

$$
\operatorname{Tr}^{s s}\left(\operatorname{Frob}_{q^{n}},\left(\mathrm{R} \Psi_{\mathcal{A}} \mathbb{Q}_{\ell}\right)_{A}\right)=\epsilon_{t(w)} \epsilon_{w} R_{w, \mathrm{t}_{\lambda}}\left(q^{n}\right)
$$

où $R_{w, \mathrm{t}(w)}$ est le polynôme $R_{x, y}$ habituel de Kazhdan-Lusztig, où $\lambda$ est l'unique élément de $P^{\vee}$ vu comme sous-groupe de $\widetilde{W}$ tel que $w=x \mathrm{t}_{\lambda}$ avec $x \in W$ un élément de groupe de Weyl vectoriel et où $\epsilon_{w}$ est le signe $(-1)^{\ell(w)}$.

Il est naturel d'essayer d'interpréter directement cette stratification en termes de variétés abéliennes.

Soit $A$ une variété abélienne de dimension $n$ définie sur un corps séparablement clos $k$ de caractéristique $p$. Par définition, le $p$-rang de $A$ est l'entier $r$ tel que $\# A[p](k)=p^{r}$ où $A[p]$ est le sous-groupes des $p$-torsions de $A$. Le $p$-rang est un entier compris entre 0 et $n$. La variété abélienne $A$ est dite ordinaire si son $p$-rang vaut $n$. Le $p$-rang ne dépend de $A$ qu'à l'isogénie près si bien qu'il définit une fonction sur l'ensemble des points géométriques de $\mathcal{A}_{s}$.

Soit $w \in \mathrm{KR}(\mu)$ vu comme un élément de groupe de Weyl affine étendu. On peut le représenter comme une matrice $2 n \times 2 n$ normalisant le tore diagonal et ayant donc exactement $2 n$ entrées non nulles, $n$ d'entre elles valant 1 et les $n$ autres valant $t$. On définit $r(w)$ comme le nombre de $t$ sur la diagonale. 
Une autre façon agréable de définir $r(w)$ est la suivante. Son image $x$ dans le groupe de Weyl vectoriel de GSp $(2 n)$ peut être considéré comme une permutation de l'ensemble $\{1, \ldots, 2 n\}$ commutant avec l'involution $(2 n, 2 n-$ $1, \cdots, 2,1)$. L'ensemble des points fixes de $x$ est en particulier stable par cette involution. Son cardinal est donc un nombre pair (puisque l'involution est sans point fixe) et vaut en fait $2 r(w)$.

Théorème 4.1 La fonction p-rang est constante sur chaque strate $\mathcal{A}_{w}$ pour tout $w \in \operatorname{KR}(\mu)$. Plus précisément, le p-rang d'un point $A \in \mathcal{A}_{w}(k)$ est égal à l'entier $r(w)$.

Démonstration. Soit $A \in \mathcal{A}(k)$ où $k$ est le corps séparablement clos de caractéristique $p$ figurant dans l'énoncé

$$
A=\left(A_{0} \stackrel{\alpha}{\longrightarrow} A_{1} \stackrel{\alpha}{\longrightarrow} \cdots \stackrel{\alpha}{\longrightarrow} A_{n}, \lambda_{0}, \lambda_{n}, \iota_{N}\right) .
$$

L'isogénie $A_{i-1} \stackrel{\alpha}{\longrightarrow} A_{i}$ induit un morphisme linéaire de rang $2 n-1$ entre les $k$-espaces $\alpha: M_{i} \rightarrow M_{i-1}$ qui sont les $\mathrm{H}_{\mathrm{DR}}^{1}$ de $A_{i}$ et $A_{i-1}$ respectivement. Ce morphisme linéaire induit par restriction un morphisme entre les sousespaces vectoriels $\omega_{i} \rightarrow \omega_{i-1}$ de $M_{i}$ et $M_{i-1}$ respectivement. Soit $H_{i}$ le noyau de l'homormorphisme $\alpha: A_{i-1} \rightarrow A_{i}$. Ce $H_{i}$ étant un groupe fini et plat de rang $p$, on peut se demander s'il est étale ou de type multiplicatif ou biconnexe. D'après de Jong [10], on sait que

1. $H_{i}$ est étale si et seulement si $\omega_{i} \rightarrow \omega_{i-1}$ est un isomorphisme, ou ce qui est équivalent $\omega_{i} \cap \operatorname{ker}\left(M_{i} \rightarrow M_{i-1}\right)=0$,

2. $H_{i}$ est de type multiplicatif si et seulement si $\omega_{i-1} \not \subset \alpha\left(M_{i}\right)$.

Ces deux conditions s'échangent clairement par dualité, $\omega_{i-1}^{\vee}$ a une intersection nulle avec $\operatorname{ker}\left(\alpha^{\vee}: M_{i-1}^{\vee} \rightarrow M_{i}^{\vee}\right)$ si et seulement si $\omega_{i-1} \not \subset \alpha\left(M_{i}\right)$. La première assertion vient du fait que l'espace des différentielles sur $H_{i}$ est $\omega_{i-1} / \alpha\left(\omega_{i}\right)$ et qu'il est nul si et seulement si $H_{i}$ est étale.

Soit $(A, \iota) \in \mathcal{W}(k)$ un relèvement de $A$ à $\mathcal{W}$. Soit $\mathcal{L}=f(\mathcal{A}, \iota) \in \mathcal{M}(k)$ dont la donnée est équivalente à un drapeau périodique de réseaux $\mathcal{L}_{i, x}$ tel que

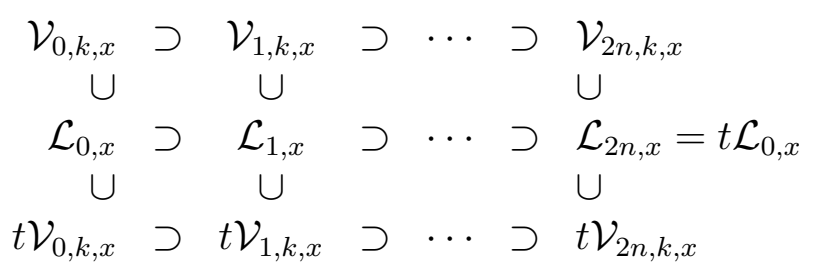

avec une condition de dualité entre $\mathcal{L}_{i, x}$ et $\mathcal{L}_{2 n-i, x}$. Les conditions 1 et 2 précédents sont alors équivalentes respectivement aux conditions suivantes

1. $\mathcal{L}_{i, x} / t \mathcal{V}_{i, k, x} \rightarrow \mathcal{V}_{i-1, k, x} / t \mathcal{V}_{i-1, k, x}$ est injectif, 
2. $\mathcal{L}_{i-1, x} \not \subset \mathcal{V}_{i, k, x}$

Ces conditions étant invariantes par rapport à l'action de $I$, le type (étale, multiplicatif ou biconnexe) de $H_{i}$ ne dépend donc que de l'orbite de $I$. Il s'ensuit que le $p$-rang de $A$ est constant sur chaque strate $\mathcal{A}_{w}$.

Soient maintenant $\mathcal{L}_{i, x}=w \mathcal{V}_{i, k, v}$ pour tout $i=0, \ldots, 2 n$ avec $w \in \operatorname{KR}(\mu)$. Les relèvements de $w$ dans $\operatorname{GL}\left(2 n, \mathbb{F}_{p}((t))\right)$ ont été choisis de sorte que $w$ stabilise l'ensemble des vecteurs de la forme $\left\{t^{m} e_{j}\right\}$. Les chaînes de réseaux $\left(\mathcal{L}_{i, x}=w \mathcal{V}_{i, k, v}\right)_{i}$ jouissent donc de la propriété suivante : pour tout $i=1, \ldots, n$, il existe un unique vecteur parmi les ceux de la forme $t^{m} e_{j}$, qui appartient à $\mathcal{L}_{i-1, x}$, mais qui n'appartient pas à $\mathcal{L}_{i, x} ;$ de plus ce vecteur est $w\left(e_{i}\right)$. Cette observation permet de démontrer que la condition 1 est équivalente à $w\left(e_{i}\right)=t e_{i}$; la condition 2 est équivalente à $w\left(e_{i}\right)=e_{i}$. En effet :

1. Le noyau du morphisme $\mathcal{V}_{i, k, x} / t \mathcal{V}_{i, k, x} \rightarrow \mathcal{V}_{i-1, k, x} / t \mathcal{V}_{i-1, k, x}$ est un $k$-espace vectoriel de dimension 1 engendré par l'image du vecteur $t e_{i}$. L'application $\mathcal{L}_{i, x} / t \mathcal{V}_{i, k, x} \rightarrow \mathcal{V}_{i-1, k, x} / t \mathcal{V}_{i-1, k, x}$ est injective si et seulement si $\mathcal{L}_{i, x}$ ne contient pas ce vecteur. Mais te $e_{i}$ appartient à $t \mathcal{V}_{i-1, k, x} \subset \mathcal{L}_{i-1, x}$; si de plus il n'appartient pas à $\mathcal{L}_{i, x}$, il doit être égal à $w\left(e_{i}\right)$. Donc, l'injectivité de $\mathcal{L}_{i, x} / t \mathcal{V}_{i, k, x} \rightarrow \mathcal{V}_{i-1, k, x} / t \mathcal{V}_{i-1, k, x}$ est équivalente à $w\left(e_{i}\right)=t e_{i}$.

2. Puisque $\mathcal{L}_{i-1, x} \subset \mathcal{V}_{i-1, k, x}$, la condition $\mathcal{L}_{i-1, x} \not \subset \mathcal{V}_{i, k, x}$ est satisfaite si et seulement si $e_{i} \in \mathcal{L}_{i-1, x}$. Mais $e_{i} \notin \mathcal{V}_{i, k, x}$ d'où $e_{i} \notin \mathcal{L}_{i, x}$. Donc $e_{i} \in \mathcal{L}_{i-1, x}$ si et seulement si $w\left(e_{i}\right)=e_{i}$.

Le $p$-rang étant au nombre des indices $i=1, \ldots, 2 n$ pour lesquelles la condition 1 est satisfaite, il vaut $r(w)$ le nombre de $t$ sur la diagonale dans l'expression matricielle de $w$.

Corollaire 4.2 Soit $A \in \mathcal{A}_{w}(k)$. Alors $A$ est ordinaire si et seulement si $w=$ $\mathrm{t}_{\mathrm{y} \mu}$ pour un certain $y \in W(\mathrm{GSp}(2 n))$.

Démonstration. Soit $w=\mathrm{t}_{\lambda} x$ avec $x \in W(\operatorname{GSp}(2 n))$. Suivant la seconde description du nombre $r(w)$, le $p$-rang de $A$ vaut $n$ si et seulement si $x=1$, donc $r=t_{\lambda}$. La condition de $\mu$-permissibilité entraîne alors que $\lambda=y \mu$ pour un certain $y \in W(\mathrm{GSp}(2 n))$.

Notons $\mathcal{A}^{\text {ord }}$ le lieu ordinaire de $\mathcal{A}_{s}$; on a donc montré que $\mathcal{A}^{\text {ord }}$ est la réunion des strates correspondant aux translations $\mathcal{A}_{t_{y \mu}}$. En conjonction avec le théorème de Kottwitz-Rapoport cité dans le paragraphe précédent, nous en tirons le corollaire suivant.

Corollaire 4.3 La partie ordinaire $\mathcal{A}^{\text {ord }}$ est dense dans $\mathcal{A}_{s}$. 


\section{Références}

[1] A. Beauville, Y. Laszlo. Un lemme de descente. C. R. Acad. Sci. Paris Sér. I Math. 320 (1995), no. 3, 335-340

[2] A. Genestier. Un modèle semi-stable de la variété de Siegel de genre 3 avec structures de niveau de type $\Gamma_{0}(p)$. Compositio Math. 123 (2000), no. 3, 303328.

[3] U. Goertz. On the flatness of local models for the symplectic group. Preprint math.AG/0011202.

[4] T. Haines. Test functions for Shimura varieties : The Drinfeld case. Duke Math. J., vol. 106, No. 1 (2001), 19-40.

[5] T. Haines, B.C. Ngô. Nearby cycles on local models of some Shimura varieties. Preprint 1999 math.AG/0103047.

[6] T. Haines, B.C. Ngô. Alcoves associated to special fiber of local model. Preprint 2001 math.RT/0103048.

[7] J. Humphreys. Reflection groups and Coxeter groups. Cambridge Studies in Advanced Mathematics, 29.

[8] N. Iwahori, H. Matsumoto. On some Bruhat decomposition and the structure of the Hecke rings of $p$-adic Chevalley groups. Inst. Hautes Études Sci. Publ. Math. No. 25 (1965) 5-48

[9] R. Kottwitz et M. Rapoport. Minuscule alcoves for $\mathrm{GL}_{n}$ and $G \mathrm{Sp}_{2 n}$. Manuscripta Math. 102 (2000), no. 4, 403-428

[10] A. de Jong. The moduli spaces of principally polarized abelian varieties with $\Gamma_{0}(p)$-level structure. J. Algebraic Geom. 2 (1993), no. 4, 667-688

[11] M. Rapoport. Communication privée. Mars 2001.

[12] M. Rapoport, T. Zink. Period spaces for p-divisible groups. Annals of Mathematics Studies, 141. Princeton University Press, Princeton, NJ, 1996.

B. C. Ngô :

ngo@math.univ-paris13.fr

CNRS, UMR 7539

Université Paris-Nord

Département de mathématiques

av. J.-B. Clément

93430 Villetaneuse

FRANCE

et

IHES

35 route de Chartres

91440 Bures sur Yvettes

FRANCE
A. Genestier :

Alain.Genestier@math.u-psud.fr

CNRS, UMR 8628

Université Paris-Sud

Département de mathématiques

Bâtiment 425

91405 Orsay

FRANCE 